\title{
Longitudinal-differential interferometry: direct imaging of axial superluminal phase propagation
}

\author{
Myun-Sik Kim*, Toralf Scharf, Christoph Etrich, Carsten Rockstuhl, and Hans Herzig Peter \\ ${ }^{1}$ Optics \& Photonics Technology Laboratory, Ecole Polytechnique Fédérale de Lausanne (EPFL), \\ Breguet 2, 2000 Neuchâtel, Switzerland \\ ${ }^{2}$ Institute of Condensed Matter Theory and Solid State Optics, Abbe Center of Photonics, \\ Friedrich-Schiller-Universität Jena, 07743 Jena, Germany \\ *Corresponding author: myunsik.kim@epfl.ch
}

Received October 24, 2011; accepted November 28, 2011;

posted December 2, 2011 (Doc. ID 157088); published January 19, 2012

\begin{abstract}
We introduce and demonstrate a new interferometric method called longitudinal-differential (LD) interferometry, which measures the spatially resolved phase difference of the scattered field by an object relative to the illumination. This method is combined with a high-resolution interference microscope that allows recording three-dimensional field distributions in amplitude and phase. The method is applied to study the axial phase behavior of Arago spots, an effect observable in low-Fresnel-number systems behind objects with a size comparable to the wavelength. We directly observe the initial phase delay in the Arago spot and prove that the local phase velocity exceeds the speed of light in air. Such LD phase studies are applicable not only to the Arago spot but also to other kinds of light interactions with wavelength-scale objects, e.g., photonic nanojets. (c) 2012 Optical Society of America

OCIS Codes: $\quad 050.5080,180.3170,260.1960,260.3160,120.3180,120.5050$.
\end{abstract}

The axial phase behavior of strongly confined light fields has been attracting considerable attention mainly due to anomalous and peculiar features. For example, in 1890 Gouy discovered that when a converging diffracted spherical wave passes through its focus, the axial phase exhibits an anomalous $\pi$ shift, which is named the Gouy phase shift or Gouy anomaly in honor of his discovery [1]. The origin of this phenomenon has been studied, for example, with scalar and vector diffraction theories by Wolf and co-workers [ $2-5$ and references therein] and with Berry's phase, in other words, geometric phase [6]. Such anomalous phase evolutions are observed not only in the focus of spherical waves but also in other scenarios as long as the light is spatially confined, e.g., in photonic nanojets [7] or in the spot of Arago [8]. Most interferometric studies that aim to disclose such effects imitate Gouy's original experiment [1]. The three-dimensional (3D) optical fields close to the confinement region, to the best of our knowledge, were first reported in 7 . Often, a small-wavelength approximation assuming $\lambda \ll r$ and high-Fresnel-number (FN) measuring systems with $\lambda \ll$ $r$ or $r \ll z$ are applied in the experimental studies, where $\lambda$ is the operating wavelength, $r$ is the radius of the object, and $z$ is the distance between the observation plane and the object.

The goal of this Letter is to introduce a new measurement technique called longitudinal-differential (LD) interferometry in order to investigate axial phase behaviors when light interacts with microstructures. We are especially interested in situations where objects with critical features in the order of the wavelength are studied at low-FN conditions. Such conditions were usually avoided in earlier studies to simplify the experimental efforts. We present the experimental setup and the working principle. The setup is exemplarily applied to investigate the amplitude and phase distributions of the spot of Arago and its axial phase features. Eventually, the axial phase shifts lead to on-axis local superluminal phase propagation $[\underline{8}, \underline{9}]$. This kind of superluminal wave propaga- tions in air can be also observed in Bessel beams [10]. All such information can be reliably extracted from the LD phase map.

In general, the longitudinal phase distribution provides information on the wavefront shape and spacing. The spacing is defined in $\underline{3}$ as the smallest distance between isosurfaces of the phase whose values differ by $2 \pi$. For monochromatic plane waves, this distance equals the wavelength $\lambda_{\text {eff }}$ that takes the refractive index of the propagating medium into account. The LD phase map exclusively reveals the amount of the axial phase shifts relative to such a plane wave. With this method, the change of the local phase velocity that may exceed the speed of light of a plane wave at the same frequency can be directly measured. The advantage of such method when compared to existing techniques that base on indirect imaging of interference fringes and their analysis, such as, e.g., in $\underline{8}$, is the ability to image the superluminal phase propagation directly.

For our study we use a high-resolution interference microscope (HRIM) to record the 3D amplitude and phase field distributions emerging from the object. The schematic is given in Fig. 1(a), and details of the experimental setup are reported in our previous works $[11,12]$. The direct phase measurement is based on a phase-shifting algorithm, the Schwider-Hariharan method $[13,14]$. Investigations were performed at a single wavelength of $642 \mathrm{~nm}$ (CrystaLaser, DL640-050-3). The illumination plane wave, which is normally incident upon the sample, propagates along the positive $z$ axis and is polarized in the $x$ direction.

The scanning of the sample along the optical axis, which aims to record the 3D field structures, leads to a particular observation condition for the phase field. Suppose that the object space between the sample and the objective has a refractive index of $n_{\text {upper }}$ and the space below the sample has a refractive index of $n_{\text {lower }}$, as depicted in Fig. 1(b). The variation of the optical path length (OPL) in the object arm is then given as 


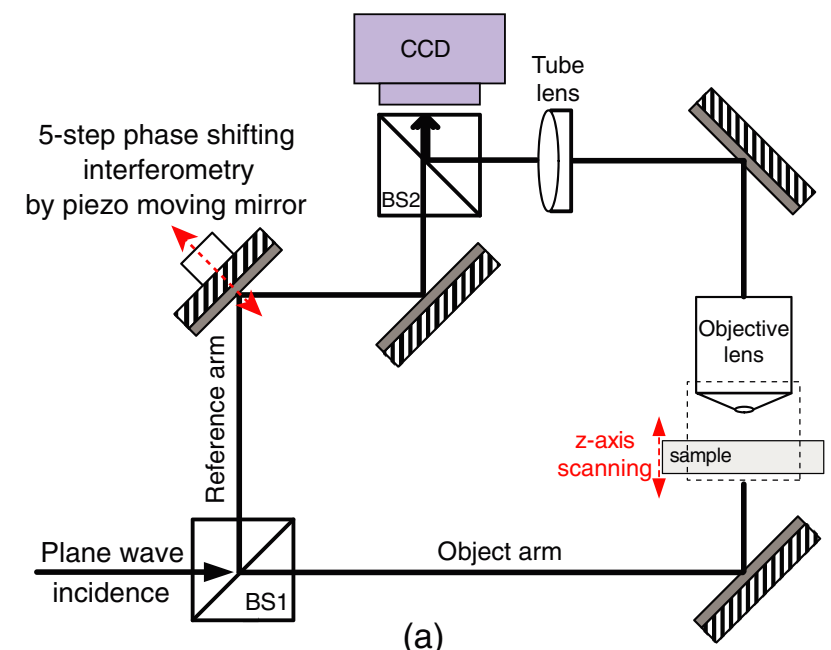

(a)

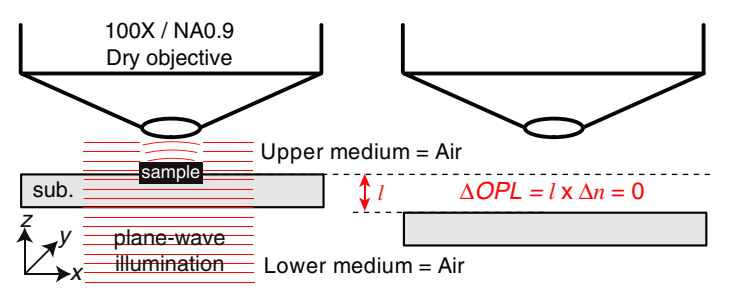

(b)

Fig. 1. (Color online) (a) Schematic of the HRIM system and (b) close-up near the sample, which shows the scanning situation.

$$
\Delta \mathrm{OPL}=\left(n_{\text {upper }}-n_{\text {lower }}\right) \cdot l,
$$

where $l$ is the scanning length along the $z$ axis. If the upper and lower medium are the same, i.e., air, then $\Delta$ OPL equals zero due to the vanishing index difference $\Delta n=0$. This means that, while scanning the sample along the $z$ axis, the phase of a plane wave remains constant. If the plane wave is perturbed due to scattering by an object, the deviation from that constant phase is measured in a self-referential manner. This eventually leads to the $\mathrm{LD}$ phase map.

A graphical representation given in Fig. 1(b) shows two waves in the space between the substrate and the objective: one is part of the unperturbed plane wave illumination propagating out of the sample and the other is the perturbed wave by the sample. The $\mathrm{LD}$ phase distribution of the unperturbed plane wave appears as constant, while that of the perturbed wave is imaged as a difference. In this way, LD interferometry measures the axial phase evolution as the difference between a plane wave (self-reference wave) and a perturbed wave (object wave).

The method is applied exemplarily to prove superluminal phase velocities in the Arago spot. Such an Arago or Poisson's bright spot occurs in the geometrical shadow of an illuminated circular obstacle, e.g., a metallic sphere or disk $[15,16]$. It is a result of the constructive interference of waves diffracted at a circular symmetric edge of the obstacle. Although the 3D amplitude and phase distributions are obtained in our measurement, we restrict our attention to on-axis observation points to analyze the phase shift and wavefront spacing.
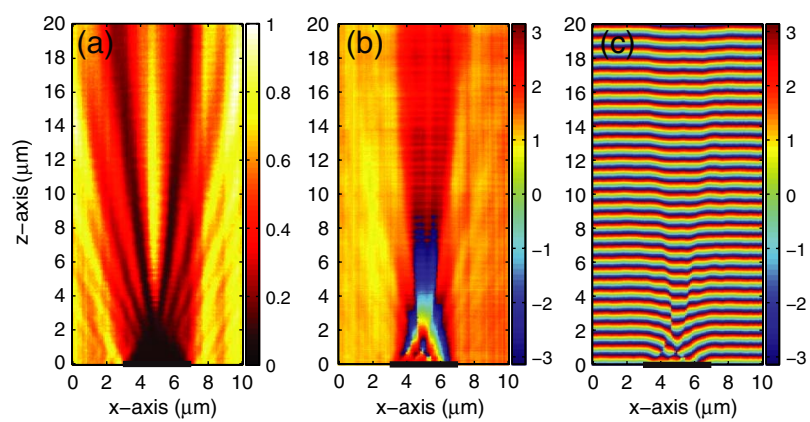

Fig. 2. (Color online) Measured longitudinal $(x-z)$ field distributions of the spot of Arago created by a $4 \mu \mathrm{m}$ metallic disk: (a) intensity, (b) LD phase, and (c) retrieved propagating phase. The intensity is normalized. The metallic disk is placed at $z=0 \mu \mathrm{m}$.

Figure 2 shows the longitudinal slices of the measured 3D intensity and phase data recorded by the LD-mode HRIM, for the case that a plane wave illuminates at normal incidence a $4 \mu \mathrm{m}$ metallic disk from below (along the positive $z$ direction). While the intensity distribution in Fig. 2(a) does not show much information except the existence of the bright spot in the shadow of the obstacle, the phase distributions reveal more detailed features in the spatial domain, where only low intensities are detectable. The propagating plane wave far away from the disk serves as the in situ self-reference plane wave. This allows correction of the inevitable phase drifts in the scanning process. In Fig. 2(b), we observe the phase evolution, and by wrapping the constant phase of the plane wave with $2 \pi$ one can reconstruct a propagation phase map shown in Fig. 2(c). We observe a wavefront bending near the edge of the obstacle and phase singularities at a distance of $z=4 \mu \mathrm{m}$ after the disk. Moreover, irregular wavefront spacings are observed close to the obstacle.

Most notably, there is an initial phase delay at the axis just behind the disk, which originates from the optical path difference between the diffracted field and the plane wave. This delay is in the order of the disk radius $r$, which is the distance the light has to travel from its outer rim to the center of the disk. Far away from the disk at large $z$ values, the path difference between the diffracted wave and the unperturbed plane wave becomes very small and finally disappears in the far field, as demonstrated in Figs. 2(b) and 2(c) $[z=15-20 \mu \mathrm{m}]$. Because of that phase difference, superluminal wavefront propagation occurs [6]. Such phase delay and on-axis phase evolution can be simply calculated considering the OPD between the diffracted wave and the propagating plane wave. To do so, we need the path length of a ray coming from the rim of the disk that travels $\left(z^{2}+r^{2}\right)^{1 / 2}$ and the propagation distance of the plane wave that is just $z$. The optical path difference becomes

$$
\mathrm{OPD} \text { plane wave - diffracted wave }=\sqrt{z^{2}+r^{2}}-z .
$$

At the disk surface, when $z=0 \mu \mathrm{m}$, Eq. (2) confirms the initial phase delay of $r$. In order to examine the superluminal behavior, we derive the phase velocity difference $(\Delta v)$ caused by such phase delays compared with that of the plane wave by differentiating Eq. (2) with respect to time [using $z=c \cdot t$ ] and find 

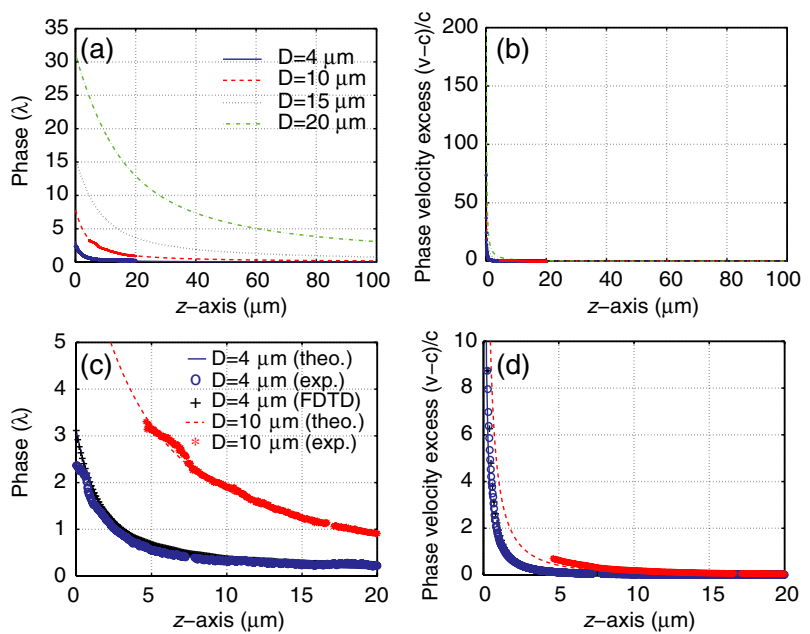

Fig. 3. (Color online) (a) On-axis LD and (b) phase velocity excess of the spot of Arago generated by different disk sizes: the solid curve for the $4 \mu \mathrm{m}$ disk, the dotted curve for the $10 \mu \mathrm{m}$ disk, the dashed curve for the $15 \mu \mathrm{m}$ disk, and the dashed-dotted curve for the $20 \mu \mathrm{m}$ disk. Open circles and asterisks represent the experimental data for 4 and $10 \mu \mathrm{m}$ disks. Close-up plots for the dashed square in (a) and (b) are (c) and (d), respectively. The FDTD results for the $4 \mu \mathrm{m}$ disk are plotted with the plus signs in (c) and (d).

$$
\Delta v=c\left(\sqrt{1+\frac{r^{2}}{z^{2}}}-1\right)
$$

with the speed of light $c$ and time $t$. Normalizing Eq. (3) leads to the phase velocity excess, $(v-c) / c$. The experimental counterpart is obtained by dividing the measured on-axis LD phase by $z$, which leads to the same result as normalizing Eq. (3) and gives

$$
\frac{\sqrt{z^{2}+r^{2}}-z}{z}=\sqrt{1+\frac{r^{2}}{z^{2}}}-1 .
$$

Figure 3 shows the experimental and theoretical phase shift and the phase velocity excess of the spot of Arago, which is generated by different disk sizes: the solid curve for the $4 \mu \mathrm{m}$ disk, the dotted curve for the $10 \mu \mathrm{m}$ disk, the dashed curve for the $15 \mu \mathrm{m}$ disk, and the dashed-dotted curve for the $20 \mu \mathrm{m}$ disk. Markers, open circles and asterisks, represent the experimental data for 4 and $10 \mu \mathrm{m}$ disks, respectively. In Fig. 3, the experimental on-axis LD phase after unwrapping and the corresponding phase velocity excess show excellent agreement with the theoretical values from Eqs. (2) and (4). Additionally, rigorous simulations based on the finite-difference time-domain method (FDTD) fully support the measurement and the analytical framework, as plotted with plus signs in Fig. 3.

For example, for the $4 \mu \mathrm{m}$ disk, Fig. 3(c) clearly shows the initial phase delay $r=2 \mu \mathrm{m}(=\sim \overline{3 \lambda})$ and the excess of the speed of light $c$ close to the disk. Because they are results of the differential data, the case of the plane wave, i.e., no phase shift and no excess of $c$, leads to 0 in both plots. Such a phase velocity excess gradually diminishes and falls to zero in the far field, for example, it exceeds just $2 \%$ of $c$ at the $z=10 \mu \mathrm{m}$ plane. When the size of the disk gets bigger, the light fields near the obstacle are difficult to measure due to the extremely low intensity. The LD phase near the $10 \mu \mathrm{m}$ disk $(z=0-4 \mu \mathrm{m})$ is therefore not properly resolved. The larger disks of 15 and $20 \mu \mathrm{m}$ diameters are only studied in theory and presented for completeness.

In conclusion, we demonstrate a new type of interferometric approach to study the axial phase behavior of light, which interacts with wavelength-scale objects. LD interferometry realized in the HRIM setup records the 3D field distributions, especially the LD phase maps. Such LD phase fields directly exhibit the phase difference compared to the plane wave propagating out of the object, which acts as the self-reference. By wrapping the constant LD phase of the self-reference plane wave in $2 \pi$, the propagating phase is also retrieved and visualizes the shape of the wavefront and the wavefront spacings. In this way, we measured the light fields of the spot of Arago at a low FN. The measured LD phase proves the initial phase delay of the disk radius $r$ and the excess of the speed of light $c$. near the disk. This explains the local superluminal wavefront propagation. Measurements show excellent agreement with the analytical values for both the axial phase shift and the phase velocity excess. These experiments verify the performance of the proposed LD interferometry, which is suitable for various kinds of micro- and nanostructures, e.g., fine gratings, dielectric microspheres, and microlenses.

The research leading to these results has received funding from the European Community's Seventh Framework Programme FP7 2007-2013 under grant agreement no. 228455.

\section{References}

1. L. G. Gouy and C. R. Hebd, Seances Acad. Sci. 110, 1251 (1890).

2. B. Richards and E. Wolf, Proc. R. Soc. London 253, 358 (1959).

3. J. T. Foley and E. Wolf, Opt. Lett. 30, 1312 (2005).

4. T. D. Visser and E. Wolf, Opt. Commun. 283, 3371 (2010).

5. X. Pang, T. D. Visser, and E. Wolf, Opt. Commun. 284, 5517 (2011).

6. D. Subbarao, Opt. Lett. 20, 2162 (1995).

7. M.-S. Kim, T. Scharf, S. Mühlig, C. Rockstuhl, and H. P. Herzig, Appl. Phys. Lett. 98, 191114 (2011).

8. M. Vasnetsov, V. Pas'ko, A. Khoroshun, V. Slyusar, and M. Soskin, Opt. Lett. 32, 1830 (2007).

9. D. Chauvat, O. Emile, M. Brunel, and A. L. Floch, Phys. Lett. A 295, 78 (2002).

10. D. Mignai, A. Ranfagni, and R. Ruggeri, Phys. Rev. Lett. 84, 4830 (2000).

11. M.-S. Kim, T. Scharf, and H. P. Herzig, Opt. Express 18, 14319 (2010).

12. M.-S. Kim, T. Scharf, S. Mühlig, C. Rockstuhl, and H. P. Herzig, Opt. Express 19, 10206 (2011).

13. J. Schwider, R. Burow, K.-E. Elssner, J. Grzanna, R. Spolaczyk, and K. Merkel, Appl. Opt. 22, 3421 (1983).

14. P. Hariharan, B. F. Oreb, and T. Eiju, Appl. Opt. 26, 2504 (1987).

15. E. Hecht, Optics (Addison-Wesley, 1987), 2nd ed., Chap. 10.

16. J. E. Harvey and J. L. Forgham, Am. J. Phys. 52, 243 (1984). 